\title{
Socio-demographic profile and prevalence of HIV infection among VCT clients in Addis Ababa
}

\author{
Antenane Korra, Mebiratu Bejiga, Solomon Tesfaye
}

\begin{abstract}
Background: VCT is an important component of the HIV/AIDS prevention and control interventions. The services are available in government non-government and HIV are health facilities. However, vary little is known about the profile and HIV prevalence among VCT clients.

Objective: The study aims at describing the socio-demographic profile of VCT clients and estimating the prevalence of HIV infection.

Methods: The data for this study were taken from VCT clients' records in the Kassanchis health center. Data were analysed using EPI-Info and SPSS.

Result: A little over half (54.1\%) of the clients were females and the clients' average age was 27.4 years (SD \pm 8.6 ). Seven in ten of the clients were never married and nearly half of the clients $(48.8 \%)$ have attended secondary school. Unemployed clients accounted for about two-thirds $(66.4 \%)$ of the total clients. The overwhelming majority of clients $(88.2 \%)$ have had sexual practices in the past. Condom use among sexually active clients was found to be low. Prevalence of HIV infection among VCT clients was estimated to be $24.5 \%$ and varied by socio-demographic characteristics of the clients. Most of the variables included in the regression model had significant effects, however, sex, employment status and a history of STIs had considerable and statistically significant influence on HIV infection than any other single factor.

Conclusion: Considering the prevailing high level of HIV infection rate among VCT clients, appropriate Behavior Change Communication (BCC) strategies need to be designed particularly focused on people with low socioeconomic status to help them lead healthy lives. In addition, strong referral linkages need to be established for the provision of care and support services. [Ethiop.J.Health Dev. 2005;19(2):109-116]
\end{abstract}

\section{Background}

Voluntary Counseling and Testing (VCT) are key components in prevention and care programs (1). The need for VCT is increasingly compelling as HIV infection rates continue to rise and; countries recognize the need for their citizens to know their sero-status as an important prevention and intervention tool (2).

In the prevention aspect, VCT helps people to learn about how HIV is transmitted, practice safer sex, get HIV test and, depending on the result, take steps to avoid becoming infected or infecting others. With in the care and support programs, HIV test results and follow up counseling mean people can be benefit from care and support services (2).

It should be emphasized that access to VCT is recognized as a critical strategy for responding to HIV/AIDS in low and middle-income as well as in high-income countries. It is thus, recommended that investing in VCT is more cost-effective and its uptake should be taken as a critical entry point to PMTCT programs (2).

There is indeed a growing demand for VCT from the general public. VCT for HIV/AIDS is included as one of the strategies HIV/AIDS prevention in the recently approved National HIV/AIDS Policy and was considered as one of the priority intervention areas with the general objective of serving as an entry point for HIV prevention by creating more personal awareness and care (3).
Ethiopia is one of the countries of the world that are most seriously affected by HIV/AIDS. The national prevalence rate of adult HIV infection is $6.6 \%$. Urban HIV prevalence rate continues to be high at $13.7 \%$ while the HIV prevalence rate for rural areas remains relatively low at $3.7 \%$. The HIV prevalence rate for Addis Ababa is estimated to be $15.6 \%$ (4). Almost $91 \%$ of the reported AIDS cases in adults are among the age group of 15 and 49 years while the highest prevalence rate is seen in the age group 15-24 years, indicating early infection.

Moreover, Ethiopian DHS revealed that only $2 \%$ of men said to have been tested for HIV, indicating that many people with HIV/AIDS in Ethiopia do not know that they are infected (5). The following are indicated in the study as factors positively affecting VCT utilization:Educational status, urban residency, masculine sex, being younger than 40 years old and unmarried status in men $(2,6)$.

A behavioral study conducted in 2002 in all regions of the country indicated that nearly two third of out-of school youth reported that they were sexually active and had sex with two or more partners in the last two years (7). Despite high level of knowledge, a significant proportion of the population, particularly the youth are at high risk of HIV infection. A significant proportions of respondents said they did not always use a condoms with 
non-regular partners, though they knew that condoms can protect from HIV/AIDS.

A study conducted from 1998-1999 in Ethiopia, on the effect of HIV-VCT on HIV incidence among a cohort of study participants indicated that HIV-VCT utilization decreases the incidence of HIV infection (8). Recent studies have shown that VCT is a cost-effective intervention for reducing HIV-related risk behaviors, particularly when applied to at risk-couples (9). VCT services are also necessary for the prevention of motherto child transmission (MTCT) of HIV and to identify women and families who may benefit from MTCT interventions. In the absence of VCT services, most women in Africa have no definitive way of knowing their HIV status until they themselves fall ill with identifiable symptoms of AIDS, or until they give birth to a baby who is diagnosed with the virus, and eventually dies from AIDS. For women identified as being HIV-positive before or during pregnancy, test related counseling can help women plan for their future and the future of their families.

HIV counseling began in Ethiopia in the late 1980s with services expanding throughout the 1990s. In the early 1990s, several national level training programs were conducted for nurses and social workers drawn from all regional hospitals and those in Addis Ababa. In addition to setting up counseling services in health institutions, the Ministry of Health $(\mathrm{MOH})$ collaborated with other NGOs like the Organization of Social Services for AIDS (OSSA), Medical Missionaries of Mary (MMM), Mother Theresa Home and others for the provision of social services to those infected (10).

Findings of a situational assessment on VCT practices in Ethiopia conducted in 2000 revealed that the demand for HIV testing was growing but service provision by government facilities was limited due to shortage of physical facilities, test kits and trained personnel. The mechanism for quality control and assurance of HIV test kit was also not well established. Only a few health facilities had full time counselors. The referral system for HIV positive individuals to care and support centers and other institutions involved in VCT services was not well developed and no system existed to monitor or evaluate VCT services. Neither the quality of counseling services provided and the efficacy of the service, nor the problem associated were assessed. However, the number and quality of VCT service seems to have increase over the past few years (10).

Integrating HIV/AIDS programming in to all projects was one of CARE Ethiopia's strategic directions given the very critical nature of the problem and its implications for CARE efforts to address household livelihood security in the population. CARE Ethiopia's Urban HIV/AIDS Prevention and Care project has established a pilot VCT center operating from within a government health institution- Kassanchis Health Center, in Nov. 2001. This VCT center was the first of its kind in terms of cost and quality (11).

Selected characteristics of VCT clients and their serostatus were recorded at the health center. An analysis of such health facility data (secondary data) particularly on VCT is limited. This study is, therefore, aiming at filling this gap and describing the socio-demographic profile of VCT clients, their reasons for getting tested and risk behavior identified as well as estimating the prevalence from HIV infection of these clients.

\section{Methods}

The data used in these analyses have come from the special records kept at the CARE-Ethiopia HIV Counseling and Testing Center at Kassanchis Health Center. Voluntary counseling and testing (VCT) data have been recorded since the inception of the service at the health center from November 2001 to February 2004 using a standardized recording format developed by the US Centers for Disease Control and Prevention (CDC). The recording formats were filled by the VCT center counselors at the time of pre and post test counseling and entered in to a computer at the project office using EPIInfo and analyzed by the SPSS statistical software package.

The study subjects are men and women clients who have visited the center for VCT services between November 2001 and February 2004. A total of 6,599 people have received the services during the specified period and records of these clients were used for analysis.

All the necessary precautions were taken to make the records confidential the client's name is not recorded on any of the formats used at the center. Uni and bi-variate analyses were made to have a primary look at of the characteristics of all the VCT clients and the HIV positive ones. These analyses call for the need for examining the case of why clients in some categories have a higher prevalence of HIV infection than others. They also provide a basis for the selection of more important variables to be included in the multivariate analysis. A multivariate analysis, mainly logistic regression, examines the importance of each variable when the influence of other variables is controlled, to identify the most important factors that affect the prevalence of HIV infection.

\section{Results}

Socio demographic profile: During the specified period of about twenty seven months, a total of 6,599 clients have received VCT services from the Kassanchis Health Center. On average there were about 244 VCT clients per month or 12 clients per day. Table 1 presents the distribution of VCT clients by some selected socio- 
demographic variables. Among the 6,599 clients a little over half $(54.1 \%)$ were females. With the overall average age of 27.4 years ( $\mathrm{SD} \pm 8.6$ ), male clients appeared to be older by about four years than their female counterparts (29.7 years vs. 25.5 years). The majority of clients were in the age range of $15-24$ years $(42.4 \%)$ and $25-34$ years $(41.1 \%)$. Seven of the total of ten clients were never married followed by currently married clients (14.4\%). Among the married and never married clients, the number of males was found to be a little higher than that of the females while among those whose marriages was dissolved (separated/divorced and widowed) the proportion of female clients is higher than males, particularly among widowed clients $(79.6 \%)$. Nearly half of the clients (48.8\%) have attained secondary school and females appeared to be less educated than males. Unemployed clients accounted for about two-thirds $(66.4 \%)$ of the total clients and $66.6 \%$ of the total unemployed clients were females.

Table 1: Percentage distribution of VCT Clients by socio-demographic variables, Addis Ababa, 2004

\begin{tabular}{lll}
\hline Variables & $\mathbf{N}$ & $\%$ \\
\hline Sex & 3032 & 45.9 \\
Male & 3567 & 54.1 \\
Female & & \\
Age & 2797 & 42.4 \\
15-24 & 2715 & 41.1 \\
$25-34$ & 916 & 13.9 \\
35-49 & 170 & 2.6 \\
50+ & SD + 8.6 & $\mathbf{2 7 . 4}$ \\
Mean age & & \\
Marital Status & 942 & 14.4 \\
Married & 4582 & 70.0 \\
Never married & 638 & 9.8 \\
Divorced/ Separated & 373 & 5.7 \\
Widowed & 14 & 0.2 \\
Others & & \\
Education & 673 & 10.3 \\
Illiterate & 197 & 3.0 \\
Able to read & 1506 & 23.1 \\
Primary & 3181 & 48.8 \\
Secondary & 953 & 14.6 \\
Tertiary & 13 & 0.2 \\
Other & & \\
Employment status & 4142 & 66.4 \\
Unemployed & 2096 & 33.6 \\
Employed & & \\
\hline
\end{tabular}

The overwhelming majority of clients $(88.2 \%)$ ever had sexual practices in the past and female clients were less likely than their male counterparts to report sexual practices $(13.6 \%$ vs. $9.7 \%)$. About $69 \%$ of the total clients had over six months suspected exposure time (time they are exposed to unsafe sex practices) and 5.8\% had no exposure (Table 2).
Condom use among sexually active VCT clients was found to be low. The vast majority $(74.7 \%)$ of the clients reported that they did not use condoms in three months time prior to testing and during last sex $(74.1 \%)$. Females are more likely than males for not using condoms $(81.2 \%$ vs. $67.4 \%$; last three months and $82.2 \%$ vs. $64.8 \%$ last sex) $(\mathrm{p}=0.000)$. One fifth of the clients had had a history of STIs and males are more likely than females in the reporting history of STI $(27.7 \%$ vs. $13.4 \%)(\mathrm{p}=0.000)$.

Sex without condom was identified as the single most important risk behavior with regard to exposure to HIV/AIDS as this was mentioned by $66.1 \%$ of the clients and a little more of the female clients $(67.8 \%)$ identified this than the males (64.1\%). Having more than one partner and blood contact were also indicated as risky behaviors by 7.1 and $5.2 \%$ of the clients respectively. There was a marked sex difference among those who mentioned having more than one partner as a risk behavior, with $9.7 \%$ for males and $4.9 \%$ for females (Table 2).

The reasons cited for seeking VCT services were diverse, but the majority (22.9\%) said they practiced risky behavior, followed those who (8.5\%) want to "plans for the future", $5.9 \%$ said it is because they have "seen" symptoms of HIV/AIDS in their body, $5.2 \%$ did for visa application and partner's risky behavior (4.2\%) (Table not shown).

According to VCT records a total of 6,584 (99.8\%) clients had their blood tested following pre-test counseling, of which about one-quarter of which (24.5\%) had test results that turned out to be HIV positive. Prevalence of HIV infection varies by the sociodemographic characteristics of the VCT clients. Table 3 presents the distribution of sero-positive clients by variables. Sero-positivity is high among females than males. According to the finding, nearly one-third of female clients $(31.5 \%)$ and $16.2 \%$ of the males were found to be HIV positive. HIV prevalence appeared to be significantly lower (15.5\%) among clients below the age of 25 than in clients at older age groups. Clients aged 3549 years exhibited higher prevalence of HIV infection (34.8\%) and also those aged 25-34 years (30.5\%). At older ages (50 and above) about one in five clients (21.4\%) had positive results.

HIV prevalence of VCT clients also varies by marital status. Ever married clients are more likely to be HIV+ than never married ones $(\mathrm{p}=0.000)$. HIV prevalence for never married clients was estimated at $17.6 \%$ as opposed to currently married $30.9 \%$. A significantly higher prevalence was found among clients whose marriages doom ended by divorce/separation (42.9\%) and death of partner (61.8\%). 
Table 2: Percentage distribution of VCT clients by sexual experience, estimated exposure time, condom use and STI history by sex, Addis Ababa, 2004

\begin{tabular}{|c|c|c|c|}
\hline \multirow[b]{2}{*}{ Category } & \multicolumn{2}{|c|}{ Sex } & \multirow[b]{2}{*}{$\begin{array}{l}\text { Total } \\
\%(n)\end{array}$} \\
\hline & $\begin{array}{l}\text { Male } \\
\%(n)\end{array}$ & $\begin{array}{l}\text { Female } \\
\%(n)\end{array}$ & \\
\hline \multicolumn{4}{|l|}{ Ever had sex } \\
\hline No & $9.7(290)$ & $13.6(482)$ & $11.8(772)$ \\
\hline Yes & $90.3(2705)$ & $86.4(3056)$ & $88.2(5761)$ \\
\hline \multicolumn{4}{|l|}{ Suspected exposure time } \\
\hline No exposure & $6.0(142)$ & $5.5(144)$ & $5.8(286)$ \\
\hline$<$ month & $10.3(242)$ & $12.7(332)$ & $11.6(574)$ \\
\hline 1 to 3 months & $12.9(302)$ & $13.6(354)$ & $13.2(656)$ \\
\hline 4 to 6 months & $12.5(294)$ & $10.1(264)$ & $11.3(558)$ \\
\hline Over 6 months & $58.3(1368)$ & $58.0(1510)$ & $58.1(2878)$ \\
\hline \multicolumn{4}{|l|}{ Condom use last 3 months } \\
\hline Never & $67.4(1723)$ & $81.2(2337)$ & $74.7(4060)$ \\
\hline Always & $24.0(614)$ & $12.2(384)$ & $17.8(968)$ \\
\hline Sometimes & $8.6(219)$ & $6.4(187)$ & $7.5(406)$ \\
\hline \multicolumn{4}{|l|}{ Used condom last sex } \\
\hline No & $64.8(1684)$ & $82.2(2428)$ & $71.1(4112)$ \\
\hline Yes & $35.2(913)$ & $17.8(526)$ & $25.9(1439)$ \\
\hline \multicolumn{4}{|l|}{ History of STI } \\
\hline No & $72.3(1922)$ & $86.6(2591)$ & $79.9(4513)$ \\
\hline Yes & $27.7(735)$ & $13.4(402)$ & $20.1(1137)$ \\
\hline Don't know & $0.3(7)$ & $0.3(10)$ & $0.3(17)$ \\
\hline \multicolumn{4}{|l|}{ Major risk behavior identified } \\
\hline Sex without condom & $64.1(1944)$ & $67.8(2420)$ & $66.1(4364)$ \\
\hline Have more than one partner & $9.7(294)$ & $4.9(175)$ & $7.1(469)$ \\
\hline Blood contact & $5.7(173)$ & $4.7(169)$ & $5.2(342)$ \\
\hline
\end{tabular}

Table 3: Percentage distribution of sero-positive clients clarified by variables, Addis Ababa, 2004

\begin{tabular}{|c|c|c|}
\hline Variables & No & $\%$ \\
\hline Total & 1612 & 24.5 \\
\hline \multicolumn{3}{|l|}{ Sex } \\
\hline Male & 491 & 16.2 \\
\hline Female & 1121 & 31.5 \\
\hline \multicolumn{3}{|l|}{ Age } \\
\hline $15-24$ & 433 & 15.5 \\
\hline $25-34$ & 825 & 30.5 \\
\hline $35-49$ & 318 & 34.8 \\
\hline $50+$ & 36 & 21.4 \\
\hline \multicolumn{3}{|l|}{ Marital Status } \\
\hline Married & 290 & 30.9 \\
\hline Never married & 807 & 17.6 \\
\hline Divorced/Separated & 273 & 42.9 \\
\hline Widowed & 230 & 61.8 \\
\hline \multicolumn{3}{|l|}{ Education } \\
\hline Illiterate & 221 & 33.0 \\
\hline Able to read & 68 & 34.5 \\
\hline Primary School & 460 & 30.6 \\
\hline Secondary School & 729 & 23.0 \\
\hline Tertiary & 108 & 11.3 \\
\hline \multicolumn{3}{|l|}{ Employment status } \\
\hline Unemployed & 1176 & 28.4 \\
\hline Employed & 366 & 17.5 \\
\hline \multicolumn{3}{|l|}{ Sexual experience } \\
\hline Never had sex & 720 & 6.5 \\
\hline Ever had sex & 1546 & 26.9 \\
\hline \multicolumn{3}{|c|}{$\begin{array}{l}\text { Condom use in the last three } \\
\text { months }\end{array}$} \\
\hline Never & 1188 & 29.3 \\
\hline Always & 157 & 16.2 \\
\hline Sometimes & 101 & 24.9 \\
\hline \multicolumn{3}{|l|}{ History of STI } \\
\hline No & 1040 & 23.1 \\
\hline Yes & 471 & 41.5 \\
\hline
\end{tabular}

The data further revealed decreasing pattern of HIV prevalence as client's education level increases. For instance among illiterate and able to read clients, HIV prevalence found to be $33 \%$ and $34.5 \%$ respectively, whereas it was only $11.3 \%$ among clients with tertiary educational level. Likewise, employed clients have lower chance to be HIV+ than the unemployed ones $(17.5 \%$ vs. $28.4 \%$ ).

Levels of HIV infection are high among clients who ever had sexual experience, who never used condom in the last three months and among those who had history of STI. Clients who ever had sex experience have more chance to be HIV+ than otherwise $(26.9 \%$ vs. $6.5 \%)$. HIV prevalence appeared to be high among clients who never used condom in three months prior to testing $(29.3 \%)$ than those who used sometimes $(24.9 \%)$ and used always $(16.2 \%)$ and the differences were statistically significant $(\mathrm{p}<0.01)$. Moreover, prevalence of HIV infection is significantly higher (41.5\%) among clients with history of STI than otherwise $(23.1 \%)$.

The univariate analysis demonstrates that the prevalence of HIV varies by demographic and socioeconomic characteristics, and most of these variables are associated with each other. Thus this analysis doesn't show independent effect of the variables. As a result the dichotomous relationship between HIV prevalence and various predictor variables was analyzed using the logistic regression method. This relationship was examined for 5,009 cases. 
The regression coefficient beta $(\beta)$ and the exponent of the coefficient of the regression estimate (the odds ratio) is shown in Table 4 . With an odds ratio of one for reference category, all other groups are compared on the basis of the reference group.

In our analyses of the prevalence of HIV infection, all the variables included in the model were found to have significant effect at $(p<0.05)$ on HIV infection except for sexual exposure in the expected directions. Although clients who had started sex are more than five times to be HIV positive as likely as clients who never had sex, the difference appeared to be statistically not significant.

However, there were two variables which had a considerable and statistically significant influence than that of any other single factor. Those variables were sex of the client and a lifetime history of STI. The coefficients in table 5 indicate that the prevalence of HIV infection greatly varies by sex. The large positive model odds ratio (2.66) for sex indicates that the prevalence of HIV infection is higher among female clients, even with controls for all the other variables. A life time history of STI has also a large and statistically significant model odds ratio (2.71), indicating that the higher level of HIV infection is exhibited among clients that have a history of STI.

Age is the other independent variable for which HIV prevalence results differ. Clients aged between 25 and 49 years have more than double chance to be HIV positive than those in the reference category (15-24 years) and its effect is statistically significant. The level of education is grouped into five categories ranging from those who are illiterate to those who have attended tertiary level education. The attendance of education only up to tertiary level has a significant effect on the prevalence of HIV infection. Those with higher educational attainment (tertiary) exhibited a lower chance to be HIV+ as compared to the illiterates.

Clients whose marriage ended because of divorce and death of a partner were also found to have a significantly high likelihood of being exposed to HIV infection than currently married clients. The employment status of clients is also important in influencing the rate of HIV infection. Currently employed clients are about $66 \%$ less likely to be infected with HIV than unemployed clients and its effect is statistically significant. Consistent condom use has also a significant effect on reducing the probability of infections.

Table 4: Logistic regression outputs: Determinants of HIV infection, Addis Ababa, 2004.

\begin{tabular}{|c|c|c|c|}
\hline Variables & Coefficient ( $\beta)$ & OR & Significance \\
\hline \multicolumn{4}{|l|}{ Sex } \\
\hline \multicolumn{4}{|l|}{ Male $^{R}$} \\
\hline Female & 0.9774 & 2.66 & 0.0000 \\
\hline \multicolumn{4}{|l|}{ Age } \\
\hline \multicolumn{4}{|l|}{$15-24^{R}$} \\
\hline $25-34$ & 0.8472 & 2.33 & 0.0000 \\
\hline $35-49$ & 0.8804 & 2.41 & 0.0000 \\
\hline $50+$ & -0.2483 & 0.78 & 0.3349 \\
\hline \multicolumn{4}{|l|}{ Marital Status } \\
\hline \multicolumn{4}{|l|}{ Married $^{\mathrm{R}}$} \\
\hline Never married & -0.1812 & 0.83 & 0.0699 \\
\hline Divorced/Separated & 0.3543 & 1.43 & 0.0046 \\
\hline Widowed & 0.9160 & 2.50 & 0.0000 \\
\hline \multicolumn{4}{|l|}{ Education } \\
\hline \multicolumn{4}{|l|}{ Illiterate $^{\mathrm{R}}$} \\
\hline Able to read & 0.2192 & 1.25 & 0.3091 \\
\hline Primary & 0.2867 & 1.33 & 0.0222 \\
\hline Secondary & 0.0878 & 1.09 & 0.4645 \\
\hline Tertiary & -0.6148 & 0.45 & 0.0002 \\
\hline \multicolumn{4}{|l|}{ Employment status } \\
\hline \multicolumn{4}{|l|}{ Unemployed $^{\mathrm{R}}$} \\
\hline Employed & -0.4097 & 0.66 & 0.0000 \\
\hline \multicolumn{4}{|l|}{ Sexual experience } \\
\hline \multicolumn{4}{|l|}{ Never had sex ${ }^{R}$} \\
\hline Ever had sex & 1.6450 & 5.18 & 0.1120 \\
\hline \multicolumn{4}{|c|}{ Condom use in three months prior to testing } \\
\hline Always & -0.4295 & 0.65 & 0.0001 \\
\hline Sometimes & -0.0942 & 0.91 & 0.4862 \\
\hline \multicolumn{4}{|l|}{ History of STI } \\
\hline $\mathrm{No}^{\mathrm{R}}$ & & & \\
\hline Yes & 0.9985 & 2.71 & 0.0000 \\
\hline
\end{tabular}




\section{Discussion}

The analyses under taken for this study tried to examine the socio-demographic characteristics of VCT clients at the center and identified the factors associated with the serostatus of clients.

The socio-demographic profile of VCT clients at Kassanchis Health Center in Addis Ababa revealed that a little over half of the clients were females in the age group of 15-34 years. Never married clients constituted a large proportion of the total number clients and more than $60 \%$ had attended secondary and above education. Unemployed clients also comprised two-thirds of the clients. This client profile appeared to be more or less similar to that of VCT clients of other health care institutions $(12,13)$. As vividly shown in the analysis, the clients were at a higher risk group of HIV infection and this was supported by a majority of those who mentioned having risky behavior as the main reason for seeking VCT services.

It was also observed that of those clients who sought VCT services, the overwhelming majority $(88.2 \%)$ ever had sexual practices in the past. This also holds true for the other institutions that provide similar services (12). This clearly shows that sexual intercourse is the single most important factor that puts people at risk of acquiring HIV. The analysis further indicated that condom use was low among the VCT clients, with only $25.3 \%$ of the clients reporting using condoms in the three months prior to testing. Females were also less likely to use condoms than their male counterparts. This is in agreement with the overall low condom utilization rate for the country ( 7 , $12,14,15)$. These differences could be attributed to women low negotiation skill on the use of condom or men's negligence and engagement in high risk sexual behavior. The perceptions that hinder people in general, and women in particular, from using condoms need to be properly addressed particularly for those who are engaged in unsafe sexual practices. Furthermore the findings of this analysis showed that one-fifth of the clients reported a life time history of STIs, a higher than that for clients of other VCT centers (12). Another finding of the analysis was clients' identification of risk behavior. According to the study subjects, sex without condom was identified as the single most important risk behavior, whereas, having more than one sex partner and making blood contacts were mentioned by a very few number of the clients $(<10 \%)$ as risk behavior. The possible plausible reason for this result could be the prevailing high promotion of condoms as a means of HIV prevention campaigns on different Media. If this is the case, concerted efforts need to be exerted to popularize all HIV prevention methods with equal vigor.

Our analyses showed a overall HIV prevalence of $24.5 \%$, in the study subjects which is significantly higher than the prevalence figure for the country's urban sentinel surveillance sites, which is $13.7 \%$ (4), Bethezatha $(18.3 \%)$ and FGAE $(15.2 \%)$ testing centers $(12,13)$. Female clients are more likely to be HIV positive than males. This pattern of female vulnerability to HIV/AIDS infection is commonly seen in many other studies perhaps due to the biological factors as well as the prevailing low socioeconomic status of women and other cultural influences.

The age pattern for HIV prevalence rates among VCT clients at the Kassanchis Health Center revealed that the prevalence is higher among older clients than younger ones. At a prevalence of $34.8 \%$, clients aged $35-49$ years exhibited the highest HIV infection rate followed by those aged $25-34$, which is $30.5 \%$, while it is estimated at $15.5 \%$ for those aged 15-24 years. The observed age pattern of HIV prevalence in this study is comparable with that for other similar studies $(12,13)$.

Prevalence of HIV infection among VCT clients has also varied due to other socio demographic variables. Like other analyses $(12,13)$, this high prevalence of HIV is also observed among those ever married, the less educated, the unemployed clients and also among clients who had started sex, who never used condoms and among those who had a history of STIs. Generally, the findings suggest that HIV affects people of low socioeconomic status and who exposed to unpleasant sexual practices. Exposures happening due to other factors than sexual practices have also contributed to HIV infection. This was demonstrated by the observed infection rate of $6.5 \%$ among clients with no sexual exposure.

According to the results of a multivariate analysis all of the socio demographic variables included in the equation controls are found to be significantly related to the prevalence of HIV infection in the expected directions. However, the sex of clients, employment status, consistent condom use and the client's lifetime history of STIs appeared to be extremely important variables in determining the prevalence of HIV infection. In such cases, the coefficients are higher in magnitude, suggesting that females, the unemployed, those who had a history of STIs and those who never used condoms were found to have a high likelihood of HIV infection than others.

In conclusion, low socioeconomic status and high risk behaviors have been found to be significantly associated with HIV infection. This indicates the need for bringing about an appropriate behavioral change communication (BCC) strategy to these particular segments of the population to help bring about meaningful behavioral change and for leading a healthy life. Considering the prevailing high HIV infection rate among VCT clients of the center $(24.5 \%)$, strong referral linkages have also to be established/strengthened with community based 
organizations (CBOs) and NGOs for care and support follow up counseling, home based care, as well as social and financial support. Other referral linkages also need to be intensified with other services of the health center for TB and STI treatment as well as for family planning services.

\section{Acknowledgement}

We wish to acknowledge the counselors the Kassanchis Health Center for efficiently filling out the form and Wrt. Hirut Tamirat for entering the data into a computer using EPI-info.

\section{References}

1. World Health Organization (WHO). HIV infected women and their families, literature review. WHO, Geneva, 2003: 4-24.

2. Center for Disease Control and Prevention (CDC). Global AIDS Program Technical Strategies -VCT. CDC, USA 2003:1-3.

3. Ethiopian AIDS Resource Center. Fact Sheet, Talking Points, Update 2003.

4. Ministry of Health. AIDS in Ethiopia, Fourth edition. MOH, Addis Ababa, Eth 2002

5. Central Statistical Authority (CSA) and ORC Macro. Demographic and Health Survey of Ethiopia 2000. CSA, Addis Ababa, Eth 2000:168-169.

6. Habte D. Assessment of the magnitude and determinants of utilization and demand for premarital VCT in civil- marriage in Addis Ababa, Eth, 2003:43-46.

7. Mitike G., et.al. HIV/AIDS behavioral surveillance Survey (BSS) Ethiopia, 2002. Round one
8. Sahilu T., Tsegaye A., Rinke T., Agonafir T., and W/Michael T. Effect of an intervention based on voluntary HIV testing and counseling on HIV incidence among ENRAP cohort study participants. A special issue on HIV/AIDS in Ethiopia. Ethiopian Medical Journal, 1999:37(1): 119.

9. Elizabeth A., Ellen G. Prevention of MTCT of HIV in Africa. Practical guidance for programs. June 2001.

10. National HIV/AIDS Council Secretariat (NACS). National Guideline for VCT in Ethiopia. October 2000.

11. CARE-Ethiopia, Urban HIV/AIDS prevention and care project. Terminal report of pilot project. April 2004.

12. Mulugeta E. Socio demographic characteristics, sexual behavior, and reasons for attending VCT service at Bethzatha VCT project. EPHA Abstract, 2003: 5 .

13. Demissie Z. Trends of HIV infection and profiles of voluntary HIV counseling and testing (VCT) service users in seven branch clinics of the Family Guidance Association of Ethiopia. EPHA Abstract, 2003: 8 .

14. Negash Y., Gebre B., Benti D., Bejiga M. A community based study on knowledge, attitudes and practice (KAP) on HIV/AIDS in Gambella Town, Western Ethiopia. Ethiop J Health Dev. 2003;17(3): 205-213.

15. Taffa N. Sexual activity of out-of-school youth, and their knowledge and attitudes about STDs and HIV/AIDS in southern Ethiopia. Ethiop. J. Health Dev. 1998; 12(1):17-22. 

